\title{
P050: Containment of methicillin resistant staphylococcus aureus (MRSA) outbreak in a neonatal intensive care unit (NICU)
}

\author{
O Eluk ${ }^{1 *}$, Y Shachor-Meyouhas' ${ }^{1}$, Y Geffen ${ }^{1}$, S Blazer ${ }^{2}$, I stein ${ }^{2}$, I Kassis ${ }^{1}$ \\ From 2nd International Conference on Prevention and Infection Control (ICPIC 2013) \\ Geneva, Switzerland. 25-28 June 2013
}

\section{Introduction}

MRSA infections in NICU are associated with significant morbidity and mortality. Early containment of outbreaks is crucial. Trials comparing different methods of screening and decolonization are lacking.

\section{Objectives}

To describe an epidemiologic and molecular investigation of MRSA outbreak in NICU.

\section{Methods}

Our NICU is a 25 bedslevel III unit. The main space has 9 beds for critically ill neonates. Two rooms serve as intermediate care ( 8 beds each). Almost 540 neonates are admitted a year. The index case was an 8 days old term baby. MRSA was isolated from his infected eye. Infection control team set an immediate investigation and emergency policy including: cohorting of $\mathrm{MRSA}^{+}$ cases, strict isolation and separate nursing team. All infants were screened for MRSA from nares, throat, axilla, groin, rectum, twice weekly, until one month after the last case discharged. Health care workers $(\mathrm{HCW})$ and parents of positive cases were screened, reeducated for infection control measures and updated daily. NICU was closed until all colonized infants were detected and isolated. Visiting was restricted. MRSA isolates were collected for molecular testing.

\section{Results}

Four colonized neonates were immediately identified by first screening. One patient was discharged and the rest were isolated in a separate room. Another infant was

'Pediatric Infectious Diseases and Control Unit, Rambam Health Care Campus, Haifa, Israel

Full list of author information is available at the end of the article identified 20 days later. The last MRSA+ neonate was discharged 3 month later. $\mathrm{HCW}$ and families screening was negative. MRSA was isolated from five infants by nasal and rectal swabs; one was detected from axilla only. Two MRSA+ patients already known in the PediatricIntensive Care Unit (PICU) located near the NICU were suspected to be the source. All NICU Isolates were identical by PFGE. The two PICU isolates were different from each other and from NICU isolates. NICU and one PICU isolates were defined as ST-5 strain by MLST. One PICU isolate was ST-627. All isolates were PVL negative and SCCmec type IV. No further cases were detected. No cases of MRSA infection occurred during the outbreak period.

\section{Conclusion}

Outbreaks of MRSA are hazardous in the NICU. Strict infection control policy and active screening may abort outbreaks.

\section{Disclosure of interest}

None declared.

\section{Author details}

${ }^{1}$ Pediatric Infectious Diseases and Control Unit, Rambam Health Care Campus, Haifa, Israel. ${ }^{2}$ Neonatal Intensive Care Unit, Rambam Health Care Campus, Haifa, Israel.

Published: 20 June 2013

\section{doi:10.1186/2047-2994-2-S1-P50}

Cite this article as: Eluk et al:: P050: Containment of methicillin resistant staphylococcus aureus (MRSA) outbreak in a neonatal intensive care unit (NICU). Antimicrobial Resistance and Infection Control 2013 2(Suppl 1): P50.

\section{C)

(c) 2013 Eluk et al; licensee BioMed Central Ltd. This is an Open Access article distributed under the terms of the Creative Commons Attribution License (http://creativecommons.org/licenses/by/2.0), which permits unrestricted use, distribution, and reproduction in any medium, provided the original work is properly cited. 